Pratt, D., Wyss, O. \& Schorlemer, W. C. (1954). J. gen. Microbiol. 11, 333-340.

\title{
A Method for Measuring Mutagenic Action
}

\author{
By D. PRATT*, O. WYSS AND W. C. SCHORLEMER
}

Department of Bacteriology, University of Texas, Austin, Texas, U.S.A.

SUMMARY: A procedure has been evolved which permits an evaluation of mutagenic activity. It appears to be relatively free of experimental bias and analyses the effect of the mutagenic agent more or less independently of selection in the growth medium. It separates the period of phenotypic expression into a distinct unit so that it may be studied separately. Its sensitivity depends on the ability to differentiate cultures containing mutants and its precision depends on the number of flasks employed for subcultures.

In a previous paper Jordan, Mefferd \& Wyss (1951) reported that broth cultures, inoculated from suspensions of Bacillus anthracis spores irradiated with ultraviolet light, contained mutants in excess of the spontaneous level, providing large enough inocula were used; suitably small inocula of irradiated organisms gave rise to cultures which were normal with respect to their concentration of mutant cells. A given irradiated suspension would produce mutant-enriched subcultures only when the inoculum of the survivors used was large enough to include at least one induced mutant. It seemed likely that a measurement of the minimal volume which produced such mutant-enriched subcultures should be useful as a measure of mutagenic activity. It is required only that the irradiated suspension be diluted to the level where, when a series of broth subcultures is inoculated from it with inocula identical in size, some of the subcultures will contain mutants in excess of the spontaneous level and others will not. When the subcultures grow to dense populations they can be assayed for content of mutants and, assuming a Poisson distribution, the number of potential mutants (cells that will produce at least some progeny of the mutant phenotype) that were in the irradiated suspension can be estimated from the dilution factor and the fraction of the subcultures which contains no more mutants than the level occurring in unirradiated controls. This method permits the phenotypic expression of all potential mutants, it depends for quantitation on a dilution procedure that will serve for estimating diverse types of mutants, it minimizes the influence of selective effects in the subculture on the final result, and it provides a sufficiently large population for detecting rare mutants when mutagenic procedures of a high degree of lethality are employed.

The experimental approach applied in this study was suggested by a similar procedure utilized by Englesberg (1952) to demonstrate the absolute increase in cells of a Pseudomonas sp. capable of utilizing itaconate after treatment with formaldehyde and by the procedure of Luria \& Delbrück (1943) for estimating the spontaneous mutation rate to phage resistance in Escherichia coli strain B.

* Present address, University of Florida, Gainesville, Florida, U.S.A. 


\section{ME'THODS}

The organism employed was the Y-53 strain of Escherichia coli K-12, a quadruple mutant differing from the parent strain in that it requires leucine, threonine, and thiamine for growth and is unable to ferment lactose (Lederberg, 1947). Suspensions of the organism were grown in brain heart infusion broth (Difco) and incubated at $37^{\circ}$ with constant shaking for $24 \mathrm{hr}$. Suitable samples of the culture were sedimented by centrifugation, washed once and resuspended in sterile water. Ten $\mathrm{ml}$. of the cell suspension containing $5 \times 10^{8}$ cells $/ \mathrm{ml}$. were placed in a sterile Petri dish and irradiated for the desired length of time by means of a G.E. Sterilamp $(15 \mathrm{w})$ at a standardized distance. The dish was agitated gently during the period of exposure.

Serial dilutions of the irradiated suspensions were prepared and from each of several dilutions, determined by a preliminary experiment to be near the end point, ten flasks containing $10 \mathrm{ml}$. of brain heart infusion broth were inoculated and incubated for $24 \mathrm{hr}$. at $37^{\circ}$. The frequency of mutants in each culture was determined by plating on suitable selective media to determine which flasks were enriched in mutants. From the first term of the Poisson distribution $\left(\boldsymbol{P}_{\mathbf{0}}=\mathrm{e}^{-\boldsymbol{M}}\right.$ or $\boldsymbol{M}=-\ln \boldsymbol{P}_{\mathbf{0}}$, where $\boldsymbol{P}_{\mathbf{0}}=$ fraction of cultures having no mutants above the spontaneous, $M=$ average number of induced mutants inoculated/flask) an estimation of the number of mutants induced by the ultraviolet treatment could be made.

Cultures were analysed for the frequency of streptomycin-resistant mutants by plating samples in quintuplicate on streptomycin assay agar containing $5 \mu \mathrm{g}$. streptomycin $/ \mathrm{ml}$; the reliability of the procedure was confirmed by assaying known mixtures of resistant and sensitive cells. Mutants capable of utilizing lactose were estimated by plating on a chemically defined medium containing lactose as the major carbon source. The components of the medium were the same as those used by Ryan (1952), except that leucine $40 \mu \mathrm{g} . / \mathrm{ml}$., threonine $40 \mu \mathrm{g} . / \mathrm{ml}$. and thiamine $1 \mu \mathrm{g} . / \mathrm{ml}$. were added. Background growth encountered by Ryan with the ML strain. of Escherichia coli was not troublesome in the case of $\boldsymbol{E}$. coli $\mathrm{Y}$-53. Recovery experiments made with known mixtures indicated that satisfactory results were obtained when the cultures were examined after $48 \mathrm{hr}$. Threonine-independent cells were assayed by plating the suspensions on the medium of Davis \& Mingioli (1950) containing leucine $40 \mu \mathrm{g} . / \mathrm{ml}$. and thiamine $1 \mu \mathrm{g} . / \mathrm{ml}$. The plates for this mutant assay were incubated at $41^{\circ}$ since this appeared to eliminate background growth. Again analysis of known mixtures indicated that satisfactory recoveries could be made.

\section{RESULTS}

In the experiment reported in Table 1 about $5.5 \%$ of the cells which were subjected to irradiation formed colonies on brain heart infusion agar. The survivors were inoculated into replicate flasks of broth at the rate of $2.2 \times 10^{6}$ viable cells/flask since preliminary experiments indicated that this number would sometimes contain induced mutants. After $24 \mathrm{hr}$. the flasks, which then contained about $5 \times 10^{9}$ cells $/ \mathrm{ml}$, were assayed for mutants. At the levels 
plated $\left(10^{8}\right.$ cells/plate) no streptomycin-resistant cultures appeared in the unirradiated population, and as can be seen from Table 1 three of the flasks inoculated with the indicated dilution of the irradiated suspension contained no mutants but seven flasks contained mutants in excess of the spontaneous level. From the $\boldsymbol{P}_{\mathbf{0}}=\mathbf{0 . 3}$ an incidence of induced mutants of $\mathbf{1 . 2}$ per $\mathbf{2 . 2} \times 10^{6}$ irradiated survivors is indicated. For the lactose mutants it appears that values larger than 70 (limits of expectation from Stevens tables: Fisher \& Yates, 1949) indicate the presence of induced mutants, and half of the flasks $\left(P_{0}=0.5\right)$ contain such mutants. For threonine independence six of the flasks contained induced mutants. In this method the actual number of the mutants on the plate has no significance except as an indication that it exceeds the spontaneous level, and therefore it need not be determined with precision. The results indicate the independent origin of the induced mutants since flasks containing increased numbers of one type of mutant may contain only the spontaneous level of the others.

The capacities of a known mixture of streptomycin-resistant and nonresistant cells, of an ultraviolet-irradiated suspension and of an untreated suspension to produce cultures containing streptomycin-resistant mutants in excess of the spontaneous level were compared. A marked similarity between the behaviour of the artificial mixture and the irradiated suspension was noted (Table 2). In both cases, cultures prepared from large inocula contained mutants above the normal frequency; however, in both, cultures prepared from smaller inocula did not. In both instances dilutions were found which when inoculated into a series of flasks would give rise to cultures only a fraction of which were enriched with mutants. By contrast cultures prepared from the untreated suspension were found to contain a uniformly low number of streptomycin-resistant mutants.

Because the method being studied depends on the recognition of cultures with mutants in numbers above the spontaneous level, an effort was made to estimate the upper limit of the frequency of streptomycin-resistant cells in cultures prepared from untreated inocula. The values found in the last three lines of Table 2 are usual, and it can be observed that they are not affected by the size of the inoculum. Of 128 such cultures started from various-sized inocula, only five have given values above $1 \times 10^{-9}$. This value has been chosen arbitrarily as a value above which the culture is considered to have streptomycin-resistant cells above the spontaneous frequency. The values for the spontaneous level were obtained by plating between 5 and $8 \times 10^{9}$ cells, the final concentration of cells $/ \mathrm{ml}$. after $24 \mathrm{hr}$. growth in brain heart infusion broth. Consequently, between 0 and 8 colonies per plate are usually found, and the zeros reported in Table 1 where $10^{8}$ cells were plated may be regarded as legitimate indication that no induced mutants were present in those flasks. When $1 \mathrm{ml}$. volumes of the untreated suspension or of the irradiated survivors were plated directly on assay agar at the concentration of streptomycin used, no colonies appeared because in the first instance the incidence of spontaneous mutants was too low and in the latter case the induced mutants required several divisions before the resistance was expressed phenotypically (Demerec, 


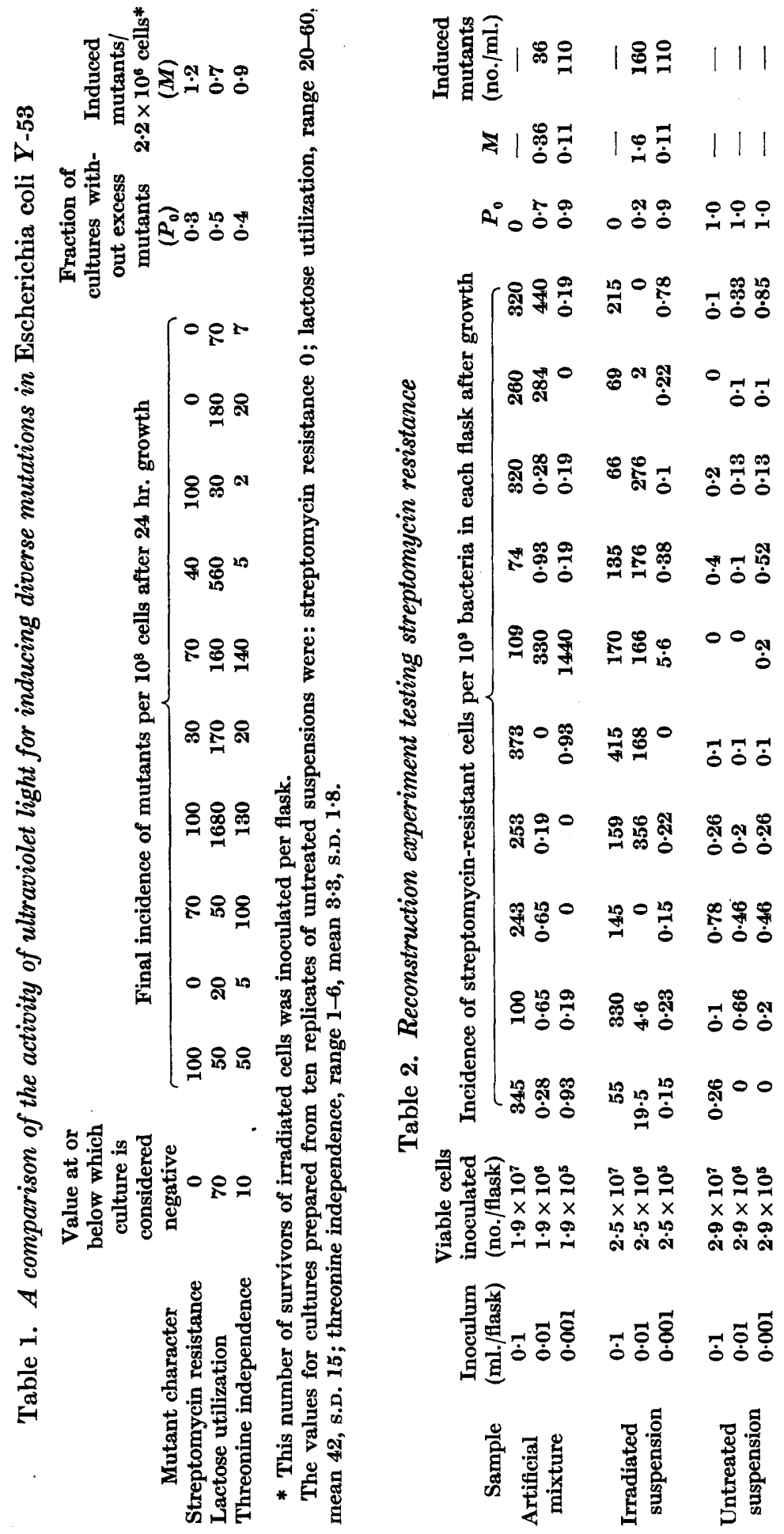


1951). However, when the $1 \mathrm{ml}$. of irradiated organism was divided into $0.1 \mathrm{ml}$. portions and inoculated into ten flasks, after a growth period all of the flasks would contain mutants at an increased level, and when only $0.01 \mathrm{ml}$. was inoculated eight out of ten subcultures were enriched in mutants. Since no mutants arose when the unirradiated culture was similarly diluted it is clearly evident without recourse to statistics that there was an absolute increase in the number of potential mutants in each millilitre of culture following irradiation.

The precision of the procedure can be increased to any desired degree by employing larger numbers of flasks. The number of streptomycin-resistant mutants in the prepared artificial mixture, estimated by plating directly on streptomycin assay agar, was $62 / \mathrm{ml}$. The values determined experimentally from two dilutions were 36 and $110 / \mathrm{ml}$, , respectively. By using the data of several dilutions and 'most probable numbers' tables as used for coliform indices, a maximum likelihood value can be obtained. The sensitivity of the procedure depends on how many bacteria must be plated to detect the spontaneous incidence of streptomycin-resistant bacteria. From the results presented it appears that at least $5 \times 10^{9}$ cells must be plated for even reasonable accuracy. Obviously, if one-tenth as many cells are plated a loss of sensitivity in the detection of positive cultures will occur since cultures with only a slight increase in the frequency of resistant cells may appear negative. However, the mechanics of the method operate against this loss of sensitivity. When $5 \times 10^{8}$ cells $/ \mathrm{ml}$. are treated with ultraviolet radiation leaving $2.5 \times 10^{8}$ cells as survivors, and $\mathbf{0 . 1} \mathrm{ml}$. of a tenfold dilution is inoculated into each of a series of flasks each flask receives $2.5 \times 10^{6}$ cells and may receive as a minimum 1 potential mutant. This indicates an initial incidence of 400 mutants $/ 10^{\circ}$ cells. If all descendants of the potential mutant are mutants and if no selection occurs during growth, the final minimal incidence should equal the initial and the plating of as few as $10^{7}$ cells should yield four colonies. Experimentally the observed minimum frequencies are considerably lower than those expected; however, most of those observed in cultures containing mutants above the spontaneous levels have been increased many-fold over the negative cultures. Consequently, a large proportion of enriched cultures can be detected by plating as few as $5 \times 10^{7}$ cells.

This property of the procedure has been utilized in devising a less laborious method for detecting enriched cultures. By placing one drop (c. 0.05 ml.) of the culture on the surface of streptomycin-containing agar, spreading over an area of 1 sq.in., and allowing it to dry, approximately $2.5 \times 10^{8}$ cells may be assayed. After incubation, streptomycin-resistant cells are readily distinguished by the development of colonies in the corresponding area on the streptomycin agar. The method was patterned after that employed by Pierce, Dubos \& Schaefer (1953) for the detection of tubercle bacilli in the various organs of mice. Comparison of the results indicated that in six experiments representing an analysis of 275 cultures, by plating $1.0 \mathrm{ml}$. of culture fluid, when only one drop of each culture was used for the analysis 132 had resistant cells in excess of spontaneous level; only six false negatives and one false positive were observed. 
Table 3. A comparison of the activity of two degrees of irradiation. for inducing streptomycin resistant mutants

\begin{tabular}{|c|c|c|c|c|c|}
\hline \multirow{2}{*}{$\begin{array}{l}\text { Period of } \\
\text { irradiation } \\
\text { (sec.) }\end{array}$} & \multicolumn{2}{|c|}{ Inoculation/flask } & \multirow{2}{*}{$\begin{array}{l}\text { Fraction of } \\
\text { flasks with- } \\
\text { out induced } \\
\text { mutants }\end{array}$} & \multirow{2}{*}{$\begin{array}{c}\text { Mutants in } \\
\text { irradiated } \\
\text { suspension } \\
\text { (no./ml.) }\end{array}$} & \multirow{2}{*}{$\begin{array}{c}\text { Induced } \\
\text { mutants } \\
\text { among } \\
\text { survivors } \\
\text { (no. } / 1^{\circ} \text { ) }\end{array}$} \\
\hline & $\begin{array}{l}\text { Volume } \\
\text { (ml.) }\end{array}$ & Viable cells & & & \\
\hline 20 & $0 \cdot 1$ & $8 \times 10^{6}$ & $0 / 10$ & - & - \\
\hline 20 & 0.01 & $8 \times 10^{5}$ & $7 / 10$ & 36 & 450 \\
\hline 20 & 0.005 & $4 \times 10^{5}$ & $8 / 10$ & 45 & 560 \\
\hline 20 & 0.001 & $8 \times 10^{4}$ & $10 / 10$ & - & - \\
\hline 40 & $0 \cdot 1$ & $9 \cdot 8 \times 10^{4}$ & $5 / 10$ & 7 & 7050 \\
\hline 40 & 0.01 & $9.8 \times 10^{3}$ & $10 / 10$ & 一 & - \\
\hline 40 & 0.005 & $4.6 \times 10^{3}$ & $10 / 10$ & - & - \\
\hline 40 & 0.001 & $9.8 \times 10^{2}$ & $10 / 10$ & 一 & 一 \\
\hline
\end{tabular}

Initial number of organisms irradiated was $2.7 \times 10^{8} / \mathrm{ml}$. The percentage survival after 20 sec. irradiation was $29 \cdot 6 \%$ and after 40 sec. irradiation was $0.363 \%$.

Using this technique of analysis, which eliminates the tedious counting of colonies since visual inspection readily reveals those spots which have colonies in excess of the spontaneous rate, the mutagenic activity of 20 and 40 sec. exposure to ultraviolet light were compared (Table 3). The results were in accord with those expected, in that the absolute number of mutants was higher after the shorter irradiation but fell as the number of survivors decreased. The ratio of mutants to survivors increased with the longer period of irradiation owing to the increments of mutation events.

\section{DISCUSSION}

The observation of Jordan et al. (1951) that the ability of irradiated anthrax spores to give rise to cultures enriched in mutants was a function of the inoculum size has been extended to another organism and other mutations. It is implicit in the method reported here that there exists in the irradiated suspension, at the time of inoculating the subculture flasks, a variable factor induced by ultraviolet light which leads to the development of excess mutants in only a fraction of a series of flasks equally inoculated from a suitable dilution. This is not affected by the procedure used in assaying for the mutants since the same method shows other flasks to have mutants only at the spontaneous level. The induced mutants exist independently of the testing procedure. The experiments demonstrate that the existence of the streptomycinresistant mutant is not conditioned by the presence of streptomycin, just as that of the threonine-deficient mutant is not conditioned by the absence of threonine or that of the lactose-positive mutant by the presence of lactose. In further support of this view is the observation that mutation in each of these three loci is independent of the others. The results are consistent with the view that the ultraviolet-irradiated suspension consists of a mixture of diverse potential mutants and non-mutant cells.

The quantitative aspects of the procedure do not depend on the absolute 
number of mutants found in the mutant assay; factors of selection and competition presumably act on the spontaneous mutants in a manner similar to their action on induced mutants, and it is necessary only that the assay procedure be sufficiently sensitive to distinguish the populations that have developed from inocula enriched in mutants. It would appear that the method should be especially useful in comparing the effect of a given mutagenic procedure for several loci in the same population.

The technique presented here is basically similar to that used by Demerec (1951) for the estimation of mutation to streptomycin resistance in Escherichia coli, strain B; in that procedure, irradiated suspensions are spread directly on the surface of nutrient agar and allowed to grow for a short time after which streptomycin is applied in a second layer of agar, the streptomycin-resistant clones grow to full colonies and can be counted. Demerec \& Cahn (1953) utilized a slightly modified procedure for the estimation of induced reversions in biochemical mutants. The suspensions treated with the mutagenic agent are plated immediately on minimal media containing a suboptimal enrichment which permits enough growth for phenotypic expression to occur. The method employed in analyzing the mutation from streptomycin dependence to independence (Bertani, 1951) is similar except that background growth in the absence of streptomycin is relied upon to allow phenotypic expression. The amount of background growth in this case depends on the concentration of streptomycin in which the cells were grown before treatment with the mutagen. In each of the last two methods the period of phentoypic expression occurs in a highly selective environment. The need for adequate procedures for estimating the quantitative and qualitative effects of mutagenic agents cannot be overestimated. It is felt that the comparison of results obtained by diverse procedures will do much to clarify the properties of bacterial cells which have been subjected to mutagenic environments.

This work was supported in part by a grant from the National Research Council Committee on Growth acting for the American Cancer Society.

\section{REFERENCES}

Bertani, G. (1951). A method for detection of mutations, using streptomycin dependence in Escherichia coli. Genetics, 36, 598.

Davis, B. D. \& Mingioli, E. S. (1950). Mutants of Escherichia coli requiring methionine or vitamin $\mathbf{B}_{12}$. J. Bact. 60, 17 .

Demerec, M. (1951). Studies of the streptomycin-resistance system of mutations in E. coli. Genetics, 36, 585.

Demerec, M. \& CAHN, E. (1953). Studies of mutability in nutritionally deficient strains of Escherichia coli I. Genetic analysis of five auxotrophic strains. J. Bact. 65, 27.

Englesberg, E. (1952). The mutagenic action of formaldehyde on bacteria. J. Bact. $63,1$.

Fisher, R. A. \& YATES, F. (1949). Statistical Tables for Biological Agricultural and Medical Research. London: Oliver and Boyd.

Jordan, W. E., Mefferd, Jun. R. B. \& Wyss, O. (1951). The radiation doseresponse curve and bacterial mutations. Science, 114, 436. 
LEDERBERG, J. (1947). Gene recombination and linked segregations in Escherichia coli. Genetics, 32, 505.

LurIA, S. E. \& DexbrǘcK, M. (1943). Mutations of bacteria from virus sensitivity to virus resistance. Genetics, 28, 491.

Pierce, C. H., Dubos, R. J. \& Schaefer, W. B. (1953). Multiplication and survival of tubercle bacilli in the organs of mice. J. exp. Med. 97, 189.

Ryan, F. J. (1952). Adaptation to use lactose in Escherichia coli. J. gen. Microbiol. 7, 69.

(Received 10 March 1954) 